\title{
Offener Brief an den Herausgeber ${ }^{1}$ ).
}

Von

\author{
Prof. Dr. A. BiedL und Dr. Max Reimer.
}

Im Bande 79 Ihres Archives, S. 158, Anmerkung, haben wir uns über einen gegen uns gerichteten Angriff von Seiten Herrn v. Cy on's wie folgt geäussert:

"Unsere erste Mittheilung ,Ueber das Vagus-Phänomen bei hohem Blutdrucke', war der Gegenstand eines heftigen Angriffes von Seiten E. v. Cyon's. Da wir in unserer Publication nicht nur die Richtigkeit der theoretischen Erörterungen, sondern auch der thatsächlichen Angaben Cyon's über die Rolle der Hypophysis beim beschriebenen Phänomen auf Grund eigener Versuche bestritten haben, konnten wir auf einen Angriff von dieser Seite wohl gefasst sein. v. Cy on hat aber in seiner Polemik die Grenzen sachlicher Erwägung nicht eingehalten, sondern Spott und Satire vorgebracht, durch welche wissenschaftliche Erkenntnisse wohl nicht gefördert werden. Da wir nun keineswegs gewillt sind, seinem Beispiele in dieser Richtung $\mathrm{zu}$ folgen, wollen wir jede eingehende Erwiderung unterlassen. Wir können dies um so mehr, als v. Cyon durch keinerlei sachliche Angaben den Nachweis erbracht hat, dass er unsere Versuche einer Nachprüfung unterzogen hätte. Indem wir die definitive Entscheidung in der discutirten Frage beruhigt der Nachprüfung von anderer Seite überlassen, betrachten wir jede weitere Discussion mit v. Cy o n unsererseits als ausgeschlossen."

In der Absicht, auf dem von uns damals eingenommenen Standpunkte auch jetzt noch zu beharren, wollen wir es unterlassen, auf eine detaillirte Kritik der von v. Cy o n neuerdings aufgestellten, von uns für unrichtig gehaltenen Behauptungen einzugehen. Dagegen scheint uns ein von v. Cyon (im 81. Bande dieses Archives, S. 272, Anmerkung) gemachter Vorschlag: wir sollten unseren

1) Die Herren A. Biedl und M. Reiner bestanden auf dieser Form der Veröffentlichung.

Der Herausgeber. 
"Grundversuch" im Beisein competenter Zeugen und unter den in unserer ersten Mittheilung angegebenen Bedingungen wiederholen, zur Feststellung der wissenschaftlichen Wahrheit geeignet und daher annehmbar.

Wir geben also hiermit die |Frklärung ab, dass wir jeder Zeit bereit sind, im Institute für allgemeine und experimentelle Pathologie in Wien "vor competenten Zeugen" folgenden Versuch, und $\mathrm{z}$ war genau so, wie wir ihn in unserer ersten Mittheilung S. 397 beschrieben haben, zu demonstriren:

„An einem durch Chloroform narkotisirten, später künstlich geathmeten Hunde wird die Membrana atlanto-occipitalis der Länge nach gespalten und dadurch der Subarachnoidalraum eröffnet. Die Oeffnung wird nach oben hin durch Resection des Os occipitale so weit vergrössert, dass das Kleinhirn etwas erhoben und dadurch die Rautengrube ganz zur Anschauung gebracht werden kann. Vor der weiteren Fortsetzung des Versuches überzeugt uns eine vorausgeschickte Injection von Nebennierextract, dass das VagusPhänomen in der gewohnten präcisen Weise auszulösen sei. Nun trennten wir die Medulla oblongata durch einen knapp oberhalb der Vaguskerne geführten Schnitt vollständig vom Hirnstamme ab und führten einen zweiten Schnitt durch die Medulla zwischen dem ersten und zweiten Halswirbel. Dadurch waren die an sich vollkommen intacten Vaguskerne zwar mit ihren zugehörigen Nerven in Verbindung, von allen anderen nervösen Beziehungen aber vollständig losgelöst. Es konnte mit Ausnahme des Depressor ${ }^{1}$ )-Reflexes, dessen Beziehung mit den Vaguskernen nicht tangirt worden war, kein Reflex auf die Kerne der Vagi ablaufen, weder vom Rückenmarke noch von irgend einem Theile des Gehirnes. Es musste also auch jene Bahn zerstört worden sein, welche die Erregungen der Hypophyse

1) Nachträgliche Anmerknng: Der Ausdruck „Depressor"-Reflex beim Hunde kann unseres Erachtens nur in dem Sinne eines im Stamme des Vago-Sympathicus ablaufenden depressorischen Reflexes verstanden werden. Der Sinn des ganzen Satzes kann also nur der sein, dass wir den anatomisch nicht isolirbaren Depressor auch nicht isolirt durchschneiden konnten, und dass demnach eine analoge, im Vagus enthaltene Bahn bezw. deren Function nicht zu eliminiren war. Nebenbei sei bemerkt, dass französische und englische Autoren (François Franck, Bradford) bei centripetaler Erregung des Vagus beim Hunde kurzweg von "Depressor-Reizung" sprechen. 
zu den Vaguskernen leiten konnte und nach Cyon, in der Umgebung der Thalami optici, vielleicht im tuber cinereum' verläuft. Und trotz alledem trat nun auf Aorten-Compression oder intravenöse Injection von Nebennierenextract das Vagus-Phänomen ebenso prägnant hervor, als wenn das Centralnervensystem vollends intact geblieben wäre.

Um nun auch die ,chemischen Wirkungen der Hypophyse 6 auszuschliessen, exstirpirten wir das gesammte vor dem ersten Schnitte liegende Gehirn und legten ein besonderes Gewicht darauf, jenen Antheil der Hypophyse noch besonders zu entfernen, der in der Sattelhöhle liegen geblieben war. Aber auch jetzt trat das VagusPhänomen, an Stärke nur wenig vermindert, in typischer und prägnanter Weise hervor. Dass auch diese Vagus-Wirkung eine centrale war, erwies die auf der Höhe des Phänomens vorgenommene Durchschneidung der Vagi. Dieselbe brachte, unter ziemlich erheblichem weiterem Ansteigen des Blutdruckes, das Phänomen sofort zum Schwinden." 